\title{
Special Issue on Mobility and Intelligence Management Technologies for Wireless and Mobile Networks
}

\author{
Ilsun You • Karl Andersson • Laurence T. Yang
}

Published online: 5 April 2014

(C) Springer Science+Business Media New York 2014

Due to the evolution and popularity of wireless and mobile technologies, mobility management has been a hot topic over the last few years. Mobile solutions allows people to get online even while moving around, thus having ubiquitous access to their preferred services anytime.

In order to optimize the overall performance of mobility management, various challenges such as location update, signaling overhead, handover latency, security, privacy, cross layer communication and so forth should be addressed. Notably, this is of great interest when the terminal networks (e.g., wireless sensor networks, body area networks, RFID, surveillance system, etc.) collect plenty of context-aware "intelligent" information (e.g., various sensory data, body signals, RFID, video/picture/multimedia) since it is a critical problem how to deal and manage this "intelligence" in an optimal way.

This special issue is composed of total eleven papers, some of which are extended from the outstanding papers presented at the 4th IEEE International Workshop on Mobility Management in the Networks of the Future World (MobiWorld 2012) and the 7th International Conference on Innovative Mobile and Internet Services in Ubiquitous Computing (IMIS2013).

The special issue starts with one paper by Yang et al. where an intelligent multi-link mechanism to handle the network selection and switching between LTE and Wi-Fi is proposed. Network throughput and switch delay are evaluated and the authors show that the network throughput can be significantly improved.

I. You $(\bowtie)$

School of Information Science, Korean Bible University, Nowon-gu, Seoul, Republic of Korea e-mail: isyou@bible.ac.kr

K. Andersson

Pervasive and Mobile Computing Laboratory, Luleå University of Technology, Skellefteå, Sweden e-mail: karl.andersson@1tu.se

L. T. Yang

Department of Computer Science, St. Francis Xavier University, Antigonish, Canada

e-mail: lyang@stfx.ca 
The second paper by Chang et al. proposes an adaptive radio interface selection for balancing loads between the UMTS and LTE/LTE-advanced interfaces according to various interference and mobility environments. Results indicate that the proposed approach outperforms other approaches in fairness, FRL, utilization and call blocking.

The third paper by Kafle et al. proposes a new distributed mobility scheme for supporting seamless mobility for hosts moving across access networks of different network-layer protocols. The proposed scheme also supports mobility of an access network itself. Experimental results from a testbed implementation demonstrate that it can achieve seamless (no packet loss) handover if overlapped wireless access networks are available.

The fourth paper by Li et al. addresses the problem of cache consistency in Locator/ID Separation Protocol networks. The authors propose a lease algorithm to realize strong consistency and describe analytical models to decide the lease duration based on the state space and control message overheads. Li et al. conclude that the proposed algorithm with an appropriate lease duration can balance the state space and the control message overhead while providing strong mapping cache consistency.

The fifth paper by Shi et al. proposes ontology-driven recommendation strategies to describe and integrate tourism resources, achieving the goal of associating user's direct needs and his/her potential preference as the context in recommendation. The authors provide a theoretical analysis and report that experiments show that the proposed approach is feasible.

The sixth paper by Saleem et al. proposes Daily Activity Monitor and LifeCare Provisioner (DALP), which is a GPS-based outdoor activities analysis system for user monitoring and lifecare provisioning. Static trajectory locations are used in order to achieve real-time and accurate outcome in tracking movement activities. The authors present a proof-of-concept prototype showing the effectiveness of the proposed solution.

The seventh paper by Kim et al. addresses the problem how multiple virtual interfaces for multi-homing hosts can support inter-technology handovers in PMIPv6 networks. The authors evaluate their proposed solution by studying the performance in terms of neighbor discovery process delay and inter-technology handover latency.

In the eighth paper, Guo et al. propose DHSR, which is a novel semantic retrieval approach for ubiquitous multimedia. The work presented provides a search engine with convenient user interfaces. Experimental results show that DHSR can search the multimedia documents reflecting users' query intent more effectively compared with traditional approaches.

The ninth paper by Movania et al. describes how on-site volume rendering can be done with GPU-enabled devices. Two high-performance volume renderers are described; singlepass GPU ray caster and fast 3D texture slicer, for both mobile and desktop platforms. The authors report that the proposed mobile $3 \mathrm{D}$ image rendering system outperforms existing approaches.

The tenth paper by Liu et al. shows how user preference heterogeneous network selection can be done in less subjective ways. Two methods are proposed, one reducing the subjectiveness and the other being totally unsubjective. A few examples are presented testing the two methods.

Finally, the eleventh paper by Zheng et al. presents EIM, which is an enforceable incentive mechanism for dynamic non-cooperative multi-radio channel allocation in wireless mobile networks. The basic idea is to relax the strong assumption of saturation network traffic, which makes the mobile terminals able to have time varying bandwidth requirements. By modeling the problem as an infinite repeated game detailed theoretical analysis proves that there exist multiple pure Nash Equilibriums (NEs) for the game, and, under certain condition, there also exist NEs which are not Pareto-Optimal. Results show that the proposed solution can improve the system throughput about $15 \%$ compared with the existed works, which can 
provide an efficient method to manage channel resource and get better ubiquitous online access to preferred mobile services.

We would like to extend special thanks to all authors as well as reviewers for their enthusiasm and dedication, which have made this special issue a reality. We would also like to thank Professor Ramjee Prasad, Editor-in-Chief of Wireless Personal Communications, for giving this valuable opportunity to us.

Finally, we hope this special issue will serve as a reference point to those readers who are interested in advanced wireless mobile communications.

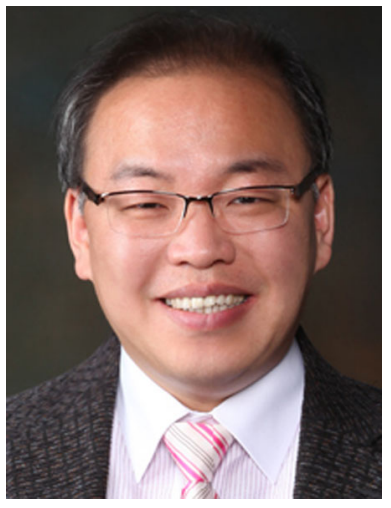

Ilsun You received his M.S. and Ph.D. degrees in Computer Science from Dankook University, Seoul, Korea in 1997 and 2002, respectively. Since March 2005, he has been an Assistant Professor in the School of Information Science at the Korean Bible University, South Korea. Dr. You served or is currently serving on the organizing or program committees of international conferences and workshops including CISIS'10-12, IMIS'07-14, MIST'09-11, MobiWorld'08-14, and so forth. Also, he has served as a guest editor for more than 15 international journals. Also, he has worked as an Editor-in-Chief for Journal of Wireless Mobile Networks, Ubiquitous Computing, and Dependable Applications (JoWUA) and Journal of Internet Services and Information Security (JISIS) while serving on the editorial boards of International Journal of Ad Hoc and Ubiquitous Computing (IJAHUC), Computing and Informatics (CAI), and International Journal of SpaceBased and Situated Computing (IJSSC). His main research interests include mobile Internet security and formal security verification. He is a senior member of the IEEE and an IFIP WG8.4 member.

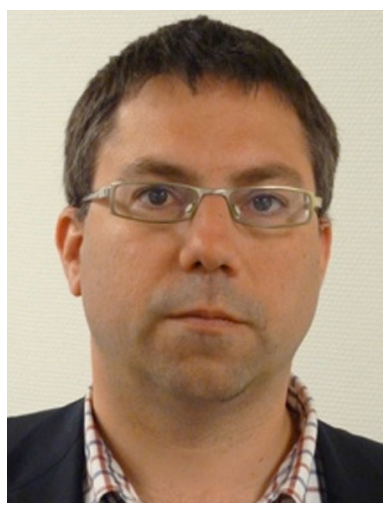

Karl Andersson received his M.Sc. degree in computer science and technology from Royal Institute of Technology, Stockholm, Sweden, in 1993. After spending more than 10 years as an IT consultant working mainly with telecom clients he returned to academia and earned his Ph.D. degree from Luleå University of Technology (LTU) in 2010 in Mobile Systems. Following his Ph.D. degree and a postdoctoral stay at Internet Real-Time Laboratory, Columbia University, New York, USA, Karl was appointed Assistant Professor of Pervasive and Mobile Computing at LTU in 2011. During Fall 2013 he was also a JSPS Fellow at National Institute of Information and Communications Technology, Tokyo, Japan. His research interests are centered around mobility management in heterogeneous networking environments, mobile eservices, and location-based services. Karl is a senior member of the IEEE. 


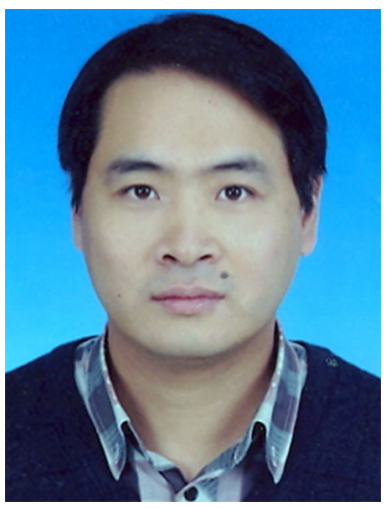

Laurence T. Yang received the B.E. degree in Computer Science and Technology from Tsinghua University, China and the Ph.D. degree in Computer Science from University of Victoria, Canada. He is currently at the Department of Computer Science, St. Francis Xavier University, Canada. His research interests include parallel and distributed computing, embedded and ubiquitous/pervasive computing, big data, cyber-physical-social systems. His research has been supported by the National Sciences and Engineering Research Council, and the Canada Foundation for Innovation. 